\title{
EL PAPEL DE LA AOI EN LA DIFUSIÓN DEL IDEAL OLÍMPICO
}

\author{
Dr. Conrado Durántez Corral \\ Presidente de la Academia Olímpica Española \\ Fecha de recepción: Junio de 2015 \\ Fecha de aceptación: Septiembre de 2015 \\ http://dx.doi.org/10.15366/citius2015.8.2.001
}

\section{Resumen:}

El presente trabajo supone la versión en español de la Conferencia que su autor presentó en la 13 Sesión Internacional para Directores de Academias Olímpicas Nacionales celebrada a inicios del mes de mayo del presente año en la Sede de la AOI en Olimpia (Grecia), invitado especialmente por los organizadores del citado evento. Si bien su contenido está publicado por la AOI en los idiomas olímpicos, la versión en español que aquí se presenta pretende ser la difusión de su contenido entre la comunidad hispanohablante.

Su contenido ofrece una descripción y reflexión sobre el impacto y el rol que ha cumplido y cumple la Academia Olímpica Internacional. Partiendo de sus antecedentes en la Grecia antigua, la AOI, heredera de la casta de los nomofilakos a la que hacía referencia su cofundador el Profesor Carl Diem, supone hoy día el indiscutible foco mundial del olimpismo. Este trabajo supone una somera revisión de los cincuenta y cuatro años del laborar de la institución, centrándose especialmente en los hitos históricos y en los logros de la AOI, como el hecho de haber supuesto la implantación de un sistema de trabajo y actuación, que ha servido de pauta de acción a las Academias Olímpicas Nacionales en la difusión y defensa del ideario olímpico entre la población de todos los países del mundo. Ha realizado y sigue desempeñando una función pedagógico-deportiva inicialmente dirigida a jóvenes estudiantes que se ha ido extendiendo a amplios sectores varios de la cultura y deporte.

Palabras clave: Academia Olímpica Internacional, Ideario olímpico, olimpismo, Movimiento Olímpico.

\section{THE ROLE OF THE INTERNATIONAL OLYMPIC ACADEMY IN THE SPREAD OF OLYMPIC IDEAL}

\begin{abstract}
:
This work includes the Spanish version of the lecture presented by the author at the 13th International Session for Directors of National Olympic Academies held in May of 2015 at the Headquarters of the IOA in Olympia (Greece). While its contents are posted by
\end{abstract}


the AOI in the official Olympic languages, the Spanish version presented here is intended as the promotion of items to the Spanish-speaking community.

This lecture provides a description and reflection on the impact and the role played by the International Olympic Academy. Starting from its origins in ancient Greece, the IOA, heir to the caste of nomofilakos to which its co-founder Professor Carl Diem refers, is today the undisputed world focus of Olympism. This work is a brief review of the fifty-four-year labor of the institution, especially focusing on the historic milestones and achievements of the IOA, like the implementation of a system of work and performance, which served as a model for the National Olympic Academies for the promotion and defense of the Olympic ideals among the population of all countries of the world. The IOA played and continues to play a sport pedagogical function. Initially aimed at young students, the institution has spread to large number of culture and sport sectors.

Keywords: International Olympic Academy, Olympic Ideology, Olympism, Olympic Movement.

Al poderoso impacto cultural del clasicismo heleno, debemos las más importantes estructuras de nuestra cultura occidental; arquitectura, estatuaria, filosofía, democracia, deporte... y olimpismo que nace como rito al gran dios nacional Zeus ${ }^{1}$, que los belicosos dorios imponen como gran patrón del politeísmo heleno y cuya morada era el Olimpo.

Pero en los tiempos modernos, Grecia ha ofrecido de nuevo al mundo dos hitos históricos transcendentes: los Juegos de Atenas de 1896 y la Academia Olímpica Internacional.

Los primeros Juegos Modernos en Atenas impulsados, basados e imbuidos por el luminoso ideario de Pierre de Coubertin, supusieron un potente aldabonazo ${ }^{2}$ a la conciencia de la humanidad alertando de que un suceso extraordinario acababa de nacer cual es el olimpismo moderno, basado fundamentalmente en su trilogía esencial de la no discriminación, la búsqueda de la paz $^{3}$ y la mejora psicofísica del ser humano a través del deporte, tendencia filosófico-deportiva que con su profundo arraigo se ha encaramado como primera fuerza sociológica del mundo. Pero además, en Grecia, en Olimpia, nació a mediados del pasado siglo la institución que ahora nos acoge, la Academia Olímpica Internacional, heredera de la antehistórica casta de los nomofilakos a la que hacía referencia su cofundador el Profesor Carl Diem ${ }^{4}$ y hoy día indiscutible foco mundial del olimpismo.

\footnotetext{
${ }^{1}$ DURÁNTEZ, Conrado: Olimpia y Los Juegos Olímpicos Antiguos, Madrid, 1975, págs.28-31, 57-59, 80-117 y 146-159.

2 DURÁNTEZ, Conrado: Academias Olímpicas Nacionales, Madrid, 1999, pág. 6

${ }^{3}$ DURÁNTEZ, Conrado: El Olimpismo moderno y su filosofía: El Ideario, Madrid 2004, pág. 24.

${ }^{4}$ DIEM, Carl: An Elis of Our Times. Meaning and Purpose of an Olympic Academy. IOA, 1961, pág.18.
} 
Cincuenta y cuatro años de ilusionado laborar de la institución, posibilitarían un extenso y fundamentado estudio de su vocacional tarea e históricos logros, a los que someramente me referiré en razón a la limitación del tiempo.

La acción de la AOI supuso el hacer despertar a nivel mundial el interés por el estudio del armazón cultural e histórico del fenómeno olímpico, extremos opacados (aún hoy día pero ya en menor medida) por el relumbrón permanente y sucesivo de las grandes proezas deportivas, que la orquesta de estruendos mediáticos, muchas veces con interesados intereses, difunden e irradian con capital primicia y transcendencia como si en un simple lance agonal y en su desenlace, estuviera en juego la dignidad o el honor de todo un pueblo.

Nunca en la vida se debe de personalizar en los relatos históricos, pero me van a permitir la excepción del caso que sirva como ejemplo al tema en estudio. Cuando yo llegué en 1961 a la inauguración en Olimpia, de una institución llamada Academia Olímpica y que nadie sabíamos en mi país de que se trataba, en aquellos momentos había finalizado mi carrera de derecho, había concurrido a un concurso nacional obteniendo plaza de juez y dos años atrás había sido preseleccionado para los Juegos Olímpicos de Roma. Pues a pesar de estas bases académico-deportivas, fue en Olimpia cuando por primera vez tuve noticia concreta por la rigurosa exposición de Paul Vialar de la vida y obra de Pierre de Coubertin, ${ }^{5}$ así como de la grandiosidad del pasado histórico de Olimpia, que me deslumbró, al ver las ruinas del Santuario en el que impresiona más lo que falta y uno se imagina, de lo que hoy queda. De entonces a hoy, la AOI ha devenido como ya en su día la calificara el histórico Profesor austriaco Josef Recla, uno de sus iniciadores, como la escuela de verano de movimiento olímpico o el Centro mundial del olimpismo.

La AOI ha implantado un sistema de trabajo y actuación, que ha servido de pauta de acción a las Academias Olímpicas Nacionales y que podríamos calificar de "académico-griego".

En algunas de las Sesiones de Olimpia, he escuchado la sorpresa de algún participante que pensaba encontrar aquí a un grupo de sesudos intelectuales que iban a tratar de olimpismo, extrañándose -indudablemente por mala información- cuando vio que el grupo determinante de participantes eran jóvenes estudiantes. Y es que la Academia de Olimpia no corresponde al patrón académico latino que se inicia a comienzo del siglo XVII, sino al esquema trazado por Platón en su escuela del saber o Academia fundada en el 387 a.C. ${ }^{6}$ y cuya mecánica de trabajo se basaba en la exposición del maestro seguida del coloquio y discusión con sus discípulos. Quizá en evitación de estos equívocos semánticos, fue lo que indujo a Pierre de Coubertin

${ }^{5}$ VIALAR, Paul: The man. The Games, IOA, 1962, pp. 15-61.

${ }^{6}$ DURÁNTEZ, Conrado: Academias Olímpicas Nacionales, Madrid, 1999, pág. 5. 
cuando hizo la propuesta de fundación de la institución al Gobierno Alemán el 16 de marzo de 1937 denominándola Centro de Estudios Olímpicos. ${ }^{7}$

La mecánica de trabajo expuesta es la que hemos seguido en mi país desde la fundación del a AOE en 1968 como primera colaboradora de la AOI y la hemos trasladado a los 27 países que conforman la Asociación Panibérica de Academias Olímpicas así como con los 34 Centros de Estudios Olímpicos que funcionan en España. La AOI en el ya pasado medio siglo de existencia, ha ganado arraigo mundial en el plano universitario y deportivo al haber tenido como norma la Eforia con su Presidente, la de invitar como exponentes de la temática a tratar, a cualificados especialistas de todos los continentes, quienes después de su intervención en Olimpia se consideran participes del quehacer de la Academia, así como los estudiantes y participantes de toda la múltiple y variada serie de cursos que hasta la fecha la AOI ha programado y desarrollado y que hablan con fervoroso entusiasmo de su experiencia en Olimpia. Y es que los cursos de Olimpia, amén de los conocimientos de la temática que se trata, lo más determinante en mi opinión, es la impresionante y conmovedora sensación que da el lugar, que capta y condiciona la voluntad del viajero ante el misterioso atractivo de la zona como así en su día emocionó a Pierre de Coubertin. ${ }^{8} \mathrm{Y}$ es que Olimpia, es un lugar de atractivo telúrico como algunos otros enclaves del planeta los que a través de los tiempos han sido punto de cita, concentración, veneración o culto para el ser humano. El recordado y entrañable profesor Paleologos ${ }^{9}$ retrotraía la vida histórica de Olimpia al siglo X a.C. como lugar de culto y veneración de toda una serie sucesiva de Dioses (Cronos, Gea, Rea) previa al de Zeus que lo impone la inmigración doria y que elige Olimpia como el paraje más evocador de Grecia, como así también lo valoraba el ateniense Lisias (446-380 a.C.) en la encendida retórica de sus discursos (43-2).

El periégeta Pausanias ubica en el mítico lugar de Olimpia la pugna y la lucha de los mismos dioses con los enfrentamientos entre Zeus y Cronos y la victoria de Apolo sobre Hermes en la carrera o contra Ares en el pugilato. ${ }^{10}$

La triple conjunción ambiental científica y grupal opera en el participante de Olimpia una especie de "bautismo olímpico" que le acompañará siempre.

Basándose en este previsible compromiso moral adquirido de aquel que vive, estudia y medita en Olimpia, hacía razonar a Otto Szymiszek, pilar histórico de los cursos de Olimpia y Decano de la institución durante un cuarto de siglo, que

\footnotetext{
${ }^{7}$ DIEM, Carl: Introduction in Olympic Academy Westfalendruck, Dortmund, p. 5.

${ }^{8}$ COUBERTIN, Pierre: Olimpismo, Lausana 2011, pág. 144, Memorias Olímpicas, Madrid 1965, pág. 220.

${ }^{9}$ PALEOLOGOS, Cleanthis: Birth, establishment and development of the Olympic Games, IOA, 1962, p. 131.

${ }^{10}$ PAUSANIAS: Descripción de Grecia, V, 7, 10 en Historiadores Griegos, Madrid, 1969, pág.260.
} 
la AOI necesitaba contar, en su tarea pedagógica, con oyentes y participantes fieles al ideario, inspirados investigadores y fanáticos misioneros del olimpismo. ${ }^{11}$

Otra de las decisivas contribuciones de la AOI al conocimiento de las múltiples y diversas facetas del ideario olímpico, ha sido el sistema de investigación y publicación de los diversos contenidos de la temática tratada y si bien hay una materia de permanente estudio (Coubertin, Juegos Olímpicos Antiguos y Modernos, etc.) se estableció a partir de 1967 un punto central a examen y debate para los conferenciantes intervinientes (el atleta olímpico, el dopaje, la mujer y el olimpismo, etc.) que ha integrado un cuerpo de saber único en la moderna historia olímpica, temática cuidadosamente publicada en las Actas de la AOI, los famosos "libros azules" en inglés, francés y griego que constituyen un fondo bibliográfico de excepcional valor para los investigadores en olimpismo y que se hizo eficazmente operativo para todo lo tratado hasta 1998 como consecuencia de la catalogación y sistematización realizada por nuestro colega Norbert Müller. ${ }^{12}$

Una AON es una "escuela de olimpismo" que hace por delegación del CON al que pertenece, la tarea básica de este, cual es la de la difusión de los principios olímpicos. Si el CON en cuestión no lo hiciese, no se debería llamar olímpico y sería simplemente un Comité para la Alta Competición o una institución para la inscripción de los atletas a los Juegos, pero no sería "olímpico". De ahí la importancia del funcionamiento de las AONs para el desarrollo de los cometidos pedagógicos olímpicos correspondientes a su CON, así como para una mayor eficacia coordinadora en los cometidos de la AOI.

Pero el tema a examen así objetivamente planteado, tiene hoy día una frustrante realidad. De los 205 CONs hoy oficialmente reconocidos, solo hay fundadas, según datos oficiosos, 137 AONs que documentalmente quedan reducidas a $133^{13}$ y de las cuales muchas más de la mitad son meramente nominativas.

La realidad operativa de una $\mathrm{AON}$, se mide fundamentalmente por al menos un curso o ciclo de conferencias anual (a imitación de la AOI) en la que se difunda el olimpismo y se capacite a la pareja de representantes que se ha de enviar a Olimpia. Tan elemental y básico cometido, está siendo mayoritariamente omitido. Pero en tal lamentable circunstancia, poco ha ayudado a resolver el problema la evolución normativa de la Carta Olímpica en lo pertinente a las AONs. Éstas, hallaron reflejo legal el 16 de julio de 1990 como consecuencia de la 96 Sesión del COI habida en Tokio por la que se modificó la norma 31 de la Carta ampliándola y precisándose en el punto 2-1 de la misma y como misión principal de los CONs, la

${ }^{11}$ SZYMISZEK, Otto, La Academia Olímpica Internacional y el movimiento olímpico contemporáneo. Madrid 1971. IV Curso de la Academia Olímpica Española.

${ }^{12}$ Thirty eight years of lectures 1961-1998, Lausana 1998.

${ }^{13}$ Dictionary of the National Olympic Academy. 2007 
de la difusión de los principios fundamentales del olimpismo dentro del país, así como su inclusión en los programas del educación física y del deporte, fomentando la creación de instituciones dedicadas a la educación olímpica, precisando a continuación fomentarán sobretodo la creación y las actividades de las Academias Olímpicas Nacionales. En consonancia con la redacción y con el contenido de la Carta en la versión que se mantuvo vigente hasta el 4 de julio de 2003, la AON venía a constituir la escuela oficial de difusión y enseñanza de los ideales y valores olímpicos, bien a escala universal cual es la AOI (norma 2-15) bien a nivel nacional cuando se trata del impulso y creación de las AONs por los CONs.

Pero la anterior redacción fue transformada como consecuencia de la modificación del texto de la Carta (ahora es la norma 27-2-1 vigente a partir de 2004) minimizando el protagonismo que la Academia como escuela oficial del olimpismo le había venido siendo atribuido al establecer el nuevo texto que... Los CONs estimularán la creación de instituciones encargadas de la educación olímpica como Academias Olímpicas Nacionales, Museos Olímpicos y programas culturales en relación con el Movimiento Olímpico.

De esta suerte la Academia, con un protagonismo especifico adquirido de concreta catalogación, pasa a integrarse en un grupo genérico de instituciones parecidas. ${ }^{14}$

La función pedagógico-deportiva de la AOI inicialmente dirigida a jóvenes estudiantes se ha ido extendiendo a amplios sectores varios de la cultura y deporte, habiéndose programado además de la tradicional sesión de jóvenes, la de directores y presidentes de AONs, postgraduados, educadores, periodistas, entrenadores, miembros de CONs, etc., habiéndose así fraguado un amplio parámetro a nivel mundial, de una amplitud de personas que tienen la común referencia de su experiencia en Olimpia.

En Olimpia es en donde la convivencia olímpica coubertiniana ${ }^{15}$ es de superior impacto a la pensada para los mismos Juegos, a los que una gran mayoría de atletas acuden agobiados por la presión precompetitiva y así la comunicación cordial y relajada es complicada, a diferencia de la convivencia en Olimpia, adonde todos acuden a dar algo en un interés común y los jóvenes blancos negros o amarillos, cuando se comunican, se dan cuenta de que piensan igual y son iguales y que integran

${ }^{14}$ DURÁNTEZ, Conrado: La función de las Academias Olímpicas Nacionales. V Forum Mondial Sur le Sport, la Culture et l'Education, Beijing, 22-24 de octubre de 2006.

${ }^{15}$ COUBERTIN, Pierre: Discurso de Pierre de Coubertin en el Parnaso en Atenas el 17 de noviembre de 1894 . 
"la primavera humana" coubertiniana ${ }^{16}$ ya que el mundo lo complican y lo estropeamos los adultos con nuestras ambiciones, codicias y torpes decisiones.

A través de las reiteradas recomendaciones dadas por la AOI por medio de sus cursos, se ha perfilado una orientación esencial a seguir por las AONs. Personalmente opino que la AON es la institución de la triple "D": difundir los principios olímpicos; defender el código ético de la competición y denunciar todas cuantas presiones, desviaciones y alteraciones que en el campo deportivo se puedan cometer y de hecho, y como reiteradamente se puede apreciar, se cometen. Como en su día razonaba Otto Szymiszek ${ }^{17}$ la esponsorización deportiva es buena porque con ella se allegan medios para la práctica deportiva, pero con ella corremos el riesgo que hemos introducido al mercader en el templo. En este sentido las AONs han de velar por lo que de acuerdo con lo que la Carta Olímpica expresa el objetivo del olimpismo sea poner siempre el deporte al servicio del desarrollo armónico del ser humano $^{18}$ ya que en un razonamiento humanista, si el deporte no sirve al hombre, el deporte para nada sirve.

La AOI ha realizado una histórica e invaluable tarea de pedagogía humanístico-deportiva a nivel mundial siendo admirable y admirada la extraordinaria labor realizada por la mayoría de los presidentes, directores o miembros de esta de los que especialmente guardo un gratísimo recuerdo entre otros Carl Diem, Jean Ketseas, Nikolaos Yalouris, Otto Szymiszek, Nissiotis, Cleanthis Paleologos, etc. Siendo de agradecer especialmente la decisiva acción de los actuales mandos de la AOI que se mantienen incólumes en su misión pese a las agudas y adversas circunstancias económicas presentes.

Señor Presidente y estimados colegas: Nuestras función con la Academia Olímpica se me antoja que en el contexto social es equivalente al volumen de voz e influencia que tenía en el cuento de Carlo Collodi pepito grillo, el grillito que era la conciencia de Pinocho, el gran muñeco de madera. Igualmente nuestra voz es tenue y chiquita, como la de él frecuentemente opacada por los estruendosos medios publicitarios de una sociedad de consumo de éticas frecuentemente amorales. Pero si nuestra pequeña voz un día se extinguiese ya no quedaría ninguna igual o parecida. De ahí que tengamos que seguir manteniéndonos con nuestra pequeña voz, pero claros y firmes.

${ }^{16}$ COUBERTIN, Pierre: Memorias olímpicas Madrid, 1965, pág. 81

${ }^{17}$ SZYMISZEK, Otto: The Olympic Ideal in the service of mankind, AOI, 1965, p. 47.

${ }^{18}$ Carta Olímpica: Principios Fundamentales-2. 


\section{Bibliografía}

- COI. (2011). Carta Olímpica. Lusana: Comité Olímpico Internacional

- COUBERTIN, Pierre: Discurso de Pierre de Coubertin en el Parnaso en Atenas el 17 de noviembre de 1894.

- COUBERTIN, Pierre: Memorias olímpicas Madrid, 1965, pág. 81

- COUBERTIN, Pierre: Olimpismo, Lausana 2011, pág. 144, Memorias Olímpicas, Madrid 1965, pág. 220.

- National Olympic Academy. (2007). Dictionary of the National Olympic Academy.

- DIEM, C. (1961). An Elis of Our Times. Meaning and Purpose of an Olympic Academy. Athens: International Olympic Academy.

- DIEM, C. (sf). Introduction in Olympic Academy. Westfalendruck, Dortmund.

- DURÁNTEZ, C. (1999). Academias Olímpicas Nacionales. Madrid: Academia Olímpica Española.

- DURÁNTEZ, C. (2004). El Olimpismo moderno y su filosofía: El Ideario, Madrid: Academia Olímpica Española.

- DURÁNTEZ, C. (2006). La función de las Academias Olímpicas Nacionales. V Forum Mondial Sur le Sport, la Culture et l'Education, Beijing. 22-24 de octubre de 2006.

- DURÁNTEZ, C. (1975). Olimpia y Los Juegos Olímpicos Antiguos. Madrid: Academia Olímpica Española.

- PALEOLOGOS, C. (1962). Birth, establishment and development of the Olympic Games. Athens: International Olympic Academy.

- PAUSANIAS. (1969). Descripción de Grecia, V, 7, 10 en Historiadores Griegos. Madrid.

- SZYMISZEK, O. (1971). La Academia Olímpica Internacional y el movimiento olímpico contemporáneo. Madrid, IV Curso de la Academia Olímpica Española. 
- SZYMISZEK, O. (1965). The Olympic Ideal in the service of mankind. Athens: International Olympic Academy.

- COI. (1998). Thirty eight years of lectures 1961-1998. Lausana: International Olympic Committee.

- VIALAR, P. (1962). The man. The Games. Athens: International Olympic Academy. 